\title{
A Weakly Majorization Inequality for Singular Values
}

\author{
Hui Quan* \\ Department of mathematics, Xiangtan University, Xiangtan411105, China
}

*Corresponding Author: Hui Quan, Department of mathematics, Xiangtan University, Xiangtan411105, China

Abstract: For the study of inequalities for singular values, it has been a research hotspot for a long time. In this paper, on the basis of $2 \times 2$ block matrix, we will study the positive semidefinite matrix ${ }^{2}\left[\begin{array}{cc}A & B \\ B^{*} & C\end{array}\right]$, and get a weakly majorization inequalityfor singular values about positive semidefiniteblock matrix.

Keywords: Inequality for Singular Values Positive Semidefinite Matrix Majorization

\section{INTRODUCTION}

In the field of matrix analysis, the techniques of block matrices playsan important role in the derivation of matrix inequalities.In recent years, the study of $2 \times 2$ block matrices, especially on the study of singular value inequalities about block matrices, has attracted extensive attention from scholars, and some very good results have also been obtained. For example, X. Zhan in reference [1] proved that $s_{j}(A-B) \leq s_{j}(A \oplus B), j=1,2, . ., n$, for positive semidefinite matrices $A, B \in M_{n}(C)$. In reference

[2], Bhatia and Kittaneh say that $2 s_{j}\left(A B^{*}\right) \leq s_{j}\left(A^{*} A+B^{*} B\right), j=1,2, \ldots, n$, for any $A, B \in M_{n}(C)$.

And recently, Audeh and Kittanehin [3] proved that if $A, B, C \in M_{n}(C)$ and satisfy that $\left[\begin{array}{ll}A & B \\ B^{*} & C\end{array}\right] \geq 0$,then $s_{j}(B) \leq s_{j}(A \oplus C), j=1,2, . ., n$.In this paper, our purpose is to study some properties of $2 \times 2$ block matrix whichmentioned above. Thus, we will get a new inequality for singular values.

\section{Preliminaries}

The matrices considered in this paper are complex matrices, and we use $M_{m, n}(C)$ to denote the space of all $m \times n$ complex matrices, when $m=n$, we use $M_{n}(C)$ to represent $M_{m, n}(C)$.For the $n \times n$ positive semi definite matrix $A$, we denote their eigen values and singular values respectively to be $\lambda_{i}(A), i=1,2, \ldots, n$ and $s_{j}(A), j=1,2, \ldots, n$, and arrange them in decreasing order by $\lambda_{1}(A) \geq \lambda_{2}(A) \geq \ldots \geq \lambda_{n}(A)$ and $s_{1}(A) \geq s_{2}(A) \geq \ldots \geq s_{n}(A)$ respectively. For the matrices $A, B, \square$ we use the symbol $A \geq B$ to represent that $A-B \square$ is positive semi definite. If $A \geq 0$ thenwe write $\lambda_{i}(A)=s_{i}(A)$.

Let the two real vectors $x=\left(x_{1}, x_{2}, \ldots, x_{n}\right)^{T}, y=\left(y_{1}, y_{2}, \ldots, y_{n}\right)^{T}$ with their components in decreasing 
order as $x_{[1]} \geq x_{[2]} \geq \ldots \geq x_{[n]}$ and $y_{[1]} \geq y_{[2]} \geq \ldots \geq y_{[n]}$.If $\sum_{i=1}^{k} x_{i} \leq \sum_{i=1}^{k} y_{i}, k=1,2, \ldots, n$, then we say that $x$ is weakly majorized by $y$, and write $x \prec_{w} y$.

Finally, the symbol $A_{1}^{*}=\left(A^{-}\right)^{T}$ denotes the conjugate transpose of matrix $A,|A|$ denotes the matrix be operated as $\left(A^{*} A\right)^{\frac{1}{2}}$.

\section{MAin Results}

It is obviously that if $A, B, C \in M_{n}(C) \square$ and satisfy $\left[\begin{array}{cc}A & B \\ B^{*} & C\end{array}\right] \geq 0$, then $A$ and $C$ are positive semi definite matrices. We hope that when $\left[\begin{array}{cc}A & B \\ B^{*} & C\end{array}\right] \geq 0$, the inequality $s_{j}(B) \leq s_{j}(A-C)$ holds. But in fact, this proposition is not necessarily true,let's look at the following example:

Let $A=\left[\begin{array}{ll}1 & 0 \\ 0 & 4\end{array}\right], C=\left[\begin{array}{ll}2 & 1 \\ 1 & 1\end{array}\right], B=\left[\begin{array}{ll}1 & 0 \\ 2 & 2\end{array}\right]$, and we can verify that $s_{2}(B)>s_{2}(A-C)$.

In spite of that, we can still get a slightly weakened conclusion. For proving convenience, we'll give the following lemmas first:

Lemma 1.1(Mirsky) If $A, B \in M_{n}(C)$, then

$\|\operatorname{diag}(s(A)-s(B))\| \leq\|A-B\|$,

and it holds for any unitary invariant norm.

Lemma1.2 If $A, B \in M_{n}(C)$, then $s(A)-s(B) \prec_{w} s(A-B)$.

Proof Wenotice that lemma 1.1 can be equivalentlyrewritten as

$|s(A)-s(B)| \prec_{w} s(A-B)$,

at the same time, the following obvious facts hold true

$s(A)-s(B) \prec_{w}|s(A)-s(B)|$,

so the lemma can be easily to get.

Lemma1.3 If $\left[\begin{array}{cc}A & B \\ B^{*} & C\end{array}\right] \geq 0$, then there exists a contractive matrix $W$ such that $B=A^{\frac{1}{2}} W C^{\frac{1}{2}}$.

Lemma1.4 If $A, B, C \in M_{n}(C)$, then we have

$s(A B C) \prec_{w} s(A) \circ s(B) \circ s(C)$.

Theorem 1Let $A, B, C \in M_{n}(C)$ and satisfy that $\left[\begin{array}{cc}A & B \\ B^{*} & C\end{array}\right] \geq 0$, if

$\lambda_{i}(A) \geq \frac{3+\sqrt{5}}{2} \lambda_{i}(C)$, then we have $s(B) \prec_{w} s(A-C)$.

Proof Fromlemma 1.2 we can know that $s(A)-s(C) \prec_{w} s(A-C)$. 
Due to $\left[\begin{array}{ll}A & B \\ B^{*} & C\end{array}\right] \geq 0$, from lemma 1.3 we can know that there exists a contractive matrix $W$ such that $B=A^{\frac{1}{2}} W C^{\frac{1}{2}}$.And then, by using lemma 1.4 , we conclude that $s(B)=s\left(A^{\frac{1}{2}} W C^{\frac{1}{2}}\right) \prec_{w} s\left(A^{\frac{1}{2}}\right) \circ s(W) \circ s\left(C^{\frac{1}{2}}\right) \prec_{w} s\left(A^{\frac{1}{2}}\right) \circ s\left(C^{\frac{1}{2}}\right)$.

At the same time, we notice that $A, C$ are positive semi definite matrices, so

$s(A)=\lambda(A), s(C)=\lambda(C)$.

When $\lambda_{i}(A) \geq \frac{3+\sqrt{5}}{2} \lambda_{i}(C)$, we can get that $\lambda_{i}(A)-\lambda_{i}(C) \geq \sqrt{\lambda_{i}(A) \lambda_{i}(C)}$,

so we can know that $s\left(A^{\frac{1}{2}}\right) \circ s\left(C^{\frac{1}{2}}\right) \prec_{w} s(A)-s(C)$,

and then $s(B) \prec_{w} s\left(A^{\frac{1}{2}}\right) \circ s\left(C^{\frac{1}{2}}\right) \prec_{w} s(A)-s(C) \prec_{w} s(A-C)$.

So, the theorem is proved

4. REMARK

In Theorem 1, if we choose $A=C, B \neq 0$, and we can see that the theorem is not true.This shows that it is necessary to add the condition $\lambda_{i}(A) \geq \frac{3+\sqrt{5}}{2} \lambda_{i}(C)$.

\section{REFERENCES}

[1] X. Zhan, On some matrix inequalities, Linear Algebra and its Applications, 376 (2004) 299-303.

[2] R. Bhatia, F. Kittaneh, Notes on matrix arithmetic-geometric mean inequalities, Linear Algaebra and its Applications, 308 (2000) 203-211.

[3] W. Audeh and F. Kittaneh.Singular value inequalities for compact operators. Linear Algebra Appl., 437:2516-2522, 2012.

[4] X. Zhan, Matrix Inequalities, LNM1790, Springer-Verlag, Berlin, 2002.

[5] A.Burqan, F.Kittaneh, Singular Value and Norm Inequalities Associated with 2 x 2 Positive Semidefinite Block Matrices, Electronic Journal of Linear Algebra, 32 (2017) 116-124.

[6] Y. Tao, More results on singular value inequalities of matrices, Linear Algebra and its Applications, 416 (2006) 724-729.

[7] X. Zhan, Singular values of differences of positive semidefinite matrices, SIAM J. Matrix Anal. Appl. 22 (2000) 819-823.

Citation: Hui Quan, "A Weakly Majorization Inequality for Singular Values", International Journal of Scientific and Innovative Mathematical Research (IJSIMR), vol. 7, no. 8, pp. 17-19, 2019. Available: DOI: http://dx.doi.org/10.20431/2347-3142.0708004

Copyright: () 2019 Authors. This is an open-access article distributed under the terms of the Creative Commons Attribution License, which permits unrestricted use, distribution, and reproduction in any medium, provided the original author and source are credited. 